EPJ Web of Conferences 44, 01004 (2013)

DOI: 10.1051/epjconf/20134401004

(C) Owned by the authors, published by EDP Sciences, 2013

\title{
Bond diluted Ising model in 2D
}

\author{
ZERGOUG Ismail ${ }^{1, \mathrm{a}}$, BOUAMRANE Rachid ${ }^{1}$, and ADDI Djamel ${ }^{1}$ \\ Laboratoire d'Etude Physique des Matériaux (LEPM) \\ USTOMB Université des Sciences et de la Technologie Mohamed Boudiaf \\ PB 1505 El Mnaouer Oran Algeria
}

\begin{abstract}
The bond diluted Ising model is studied by Monte Carlo method. The simulation is carried out on a two dimensional square lattice with missing bonds and free boundary conditions. The aim of this work is to investigate the thermodynamical properties of this model for different disorder degree parameter $\sigma$. The critical temperature is determined from the Binder cumulant and is shown to decreases as the disorder parameter $\sigma$ increases linearly.
\end{abstract}

\section{Introduction}

The 2D Ising model has been solved exactly by Onsager back on 1944 [1] and still holds nowadays an important place in the physics of phase transition and beyond. This model, in the two dimensional case, consists on a set of $\mathrm{N}$ spins located at fixed nodes. In its simplest form, each spin has two values $(+1$ or -1$)$ and interacts equally with its four nearest neighbours. With no external magnetic fields, the Hamiltonian of the system $\mathcal{H}$ is:

$$
\mathcal{H}=-\mathcal{J} \sum_{<i j>} S_{i} S_{j}
$$

The exchange coupling constant (a unique interaction factor) between nearest neighbours $<i j>$ is represented by $\mathcal{J}$ while $S_{i}$ and $S_{j}$ are the respective nearest neighbour spins.

Disordered systems have been the subject of several studies due to important effect of disorder on their thermodynamic behaviour in comparison to ordered ones [10]. Disorder can be manifested in different manner: random field [5], random interaction factors [7], site diluted [4] [8] (some of the sites are randomly occupied by either vacant lattice sites or by non magnetic atoms in the presence of magnetic ones), bond diluted [6] [3] (bonds are randomly removed from the system), etc.

In this work, we use the Binder cumulant to determine the critical temperature in the case of randomly bond diluted systems. This type of disorder was studied for the first time by D.Zobin [2] only for periodic boundary conditions. In the same context, another author has shown that the critical region becomes narrower as the concentration of bonds decreases [12].

The purpose of this paper is to report preliminary results of Monte Carlo simulations of the thermal and magnetic properties of the 2D bond diluted Ising model as done by [2] using a different approach. For now, the simulation was carried out with relatively small systems and already we have obtained interesting results worth mentioning. The value of the critical temperature at the phase transition is determined using the fourth-order cumulant Binder [9] (only free boundary conditions are considered).

\footnotetext{
a e-mail: zergoug.ismail@gmail.com
} 


\section{Model and simulation}

The Hamiltonian of the model considered here reads[2]:

$$
\mathcal{H}=-\sum_{i, j} \mathcal{J}_{i, j} S_{i} S_{j}
$$

where the exchange coupling constant $\mathcal{J}_{i, j}$ is 0 for non-nearest-neighbours and 1 or 0 with probabilities $\sigma$ or $1-\sigma$ respectively. Free boundary conditions are used, and the regular square lattice size is $L=\sqrt{N}$ (where $N$ is the total number of spins in the system). The value of the critical temperature $T_{c}$ is determinated using the Binder cumulant $U(T, L)$ defined as [9]

$$
U(T, L)=1-\frac{\left\langle M^{4}\right\rangle}{3\left\langle M^{2}\right\rangle^{2}}
$$

where $\left\langle M^{2}\right\rangle$ and $\left\langle M^{4}\right\rangle$ denote thermal averages of the second and fourth moments of the total magnetization $M$. The $U(T, L)$ versus $T$ curves for various $L$ converge toward a fixed point for large $L$ values. The reached fixed point is by definition the $T_{c}$ (Fig3).

We have performed the Monte Carlo simulation using the single spin flip algorithms [11] where we divided the equilibration process into Timesteps, the Monte Carlo steps per spin. The measurement of the thermodynamic quantities are carried out after many Timesteps, the Timeslice. To go beyond the thermodynamic equilibration stage, measurements are taken after four Timeslices and averaged at each Timeslice over a larger number of samples is done. When the average energy difference between two successively Timeslices is lower than a certain fixed error value, the simulation is stopped. This process is repeated for several temperatures $T$.

We have computed the magnetization $M$ and the specific heat $C_{v}$ in the standard manner:

$$
\begin{gathered}
M=\frac{1}{N} \sum_{i} S_{i} \\
C_{v}=N \beta^{2}\left(\left\langle E^{2}\right\rangle-\langle E\rangle^{2}\right)
\end{gathered}
$$

where $N$ is the total number of spins system, $\beta=1 / k_{B} T$ ( $T$ is the temperature and $k_{B}$ is the Boltzmann constant taken to unity here).

\section{Results and discussions}

The calculations were carried out for $\sigma=1.0,0.95,0.90,0.85$ and 0.80 using lattice sizes $L=256$, 1024 and 4096. 10000 samples were used for averaging.

The thermodynamic quantities are plotted in Fig1 and Fig2 for $L=4096$ and for various disorder degree $\sigma$ (the concentration of bond).

From Figure 3, we can get the value of the critical temperature $T_{c}$ for different degree of disorder $\sigma$ from the intersection of the cumulant for different sizes. 
1st International Conference on Numerical Physics

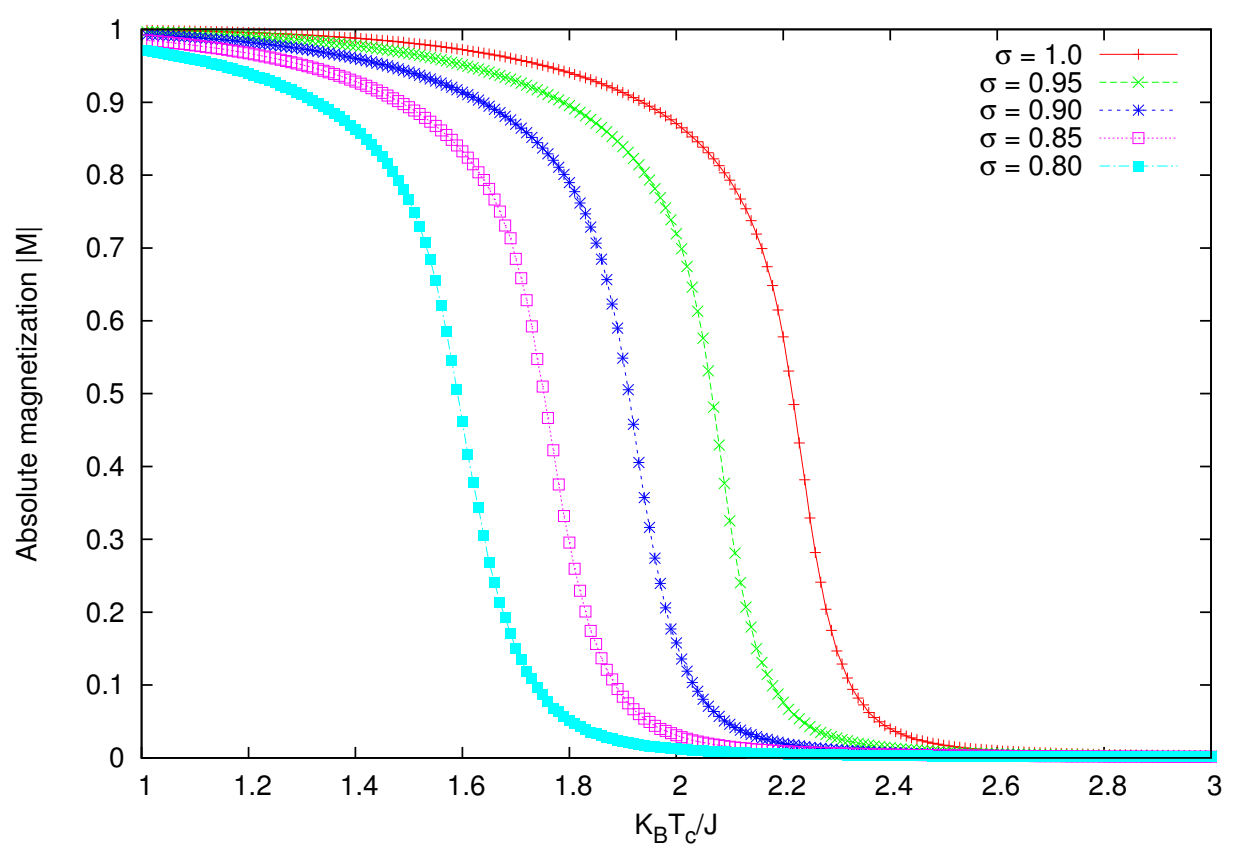

Fig. 1. The absolute magnetization $|M|$ as a function of the temperature

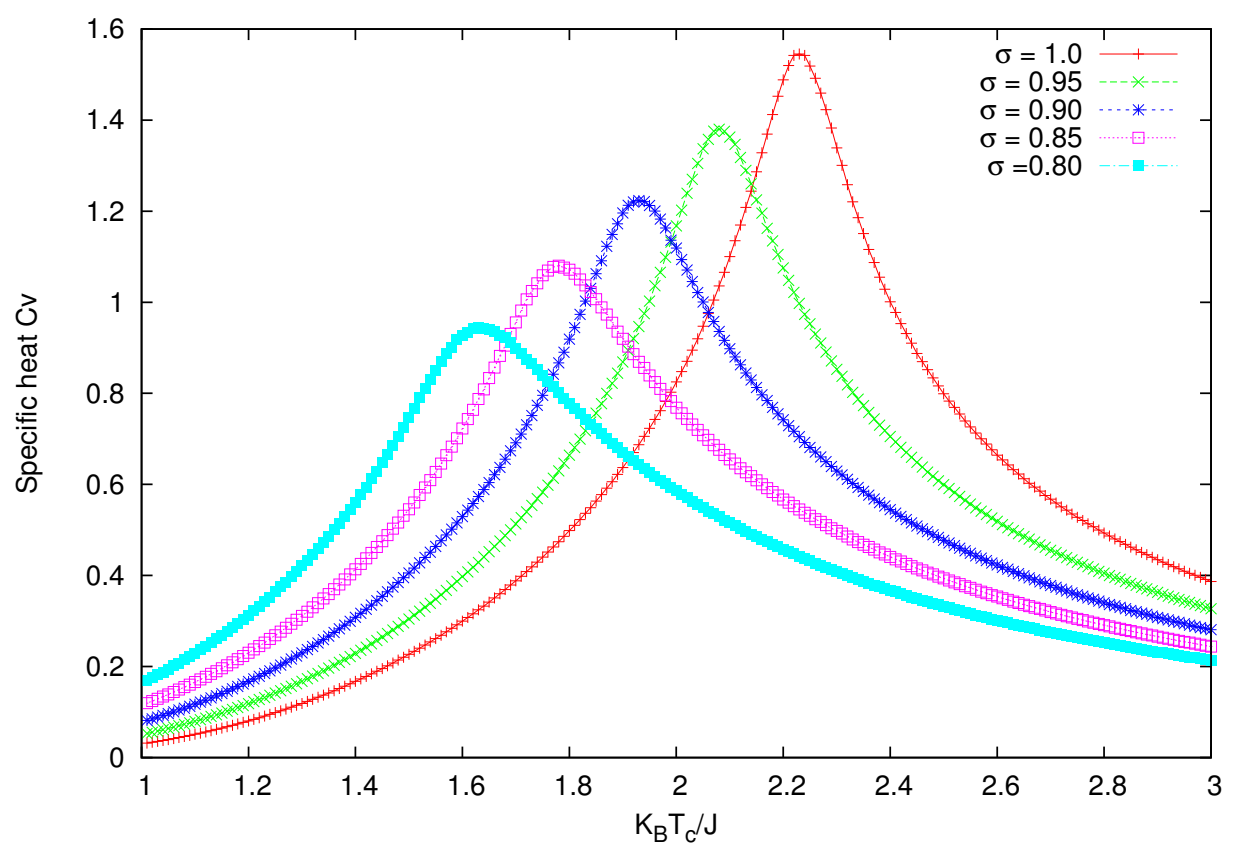

Fig. 2. The specific heat $C_{v}$ as a function of the temperature

The critical temperature $T_{c}$ has shown decrease linearly with respect to $\sigma$ (fig 4). For $\sigma=1.0$ (pure system), $T_{c}=2.2686 \pm 0.0028$ which tends to the exact critical temperature $(2 / \ln (\sqrt{2}+1)=2.26918 \ldots)$ [1] for a larger lattice sizes and samples. 


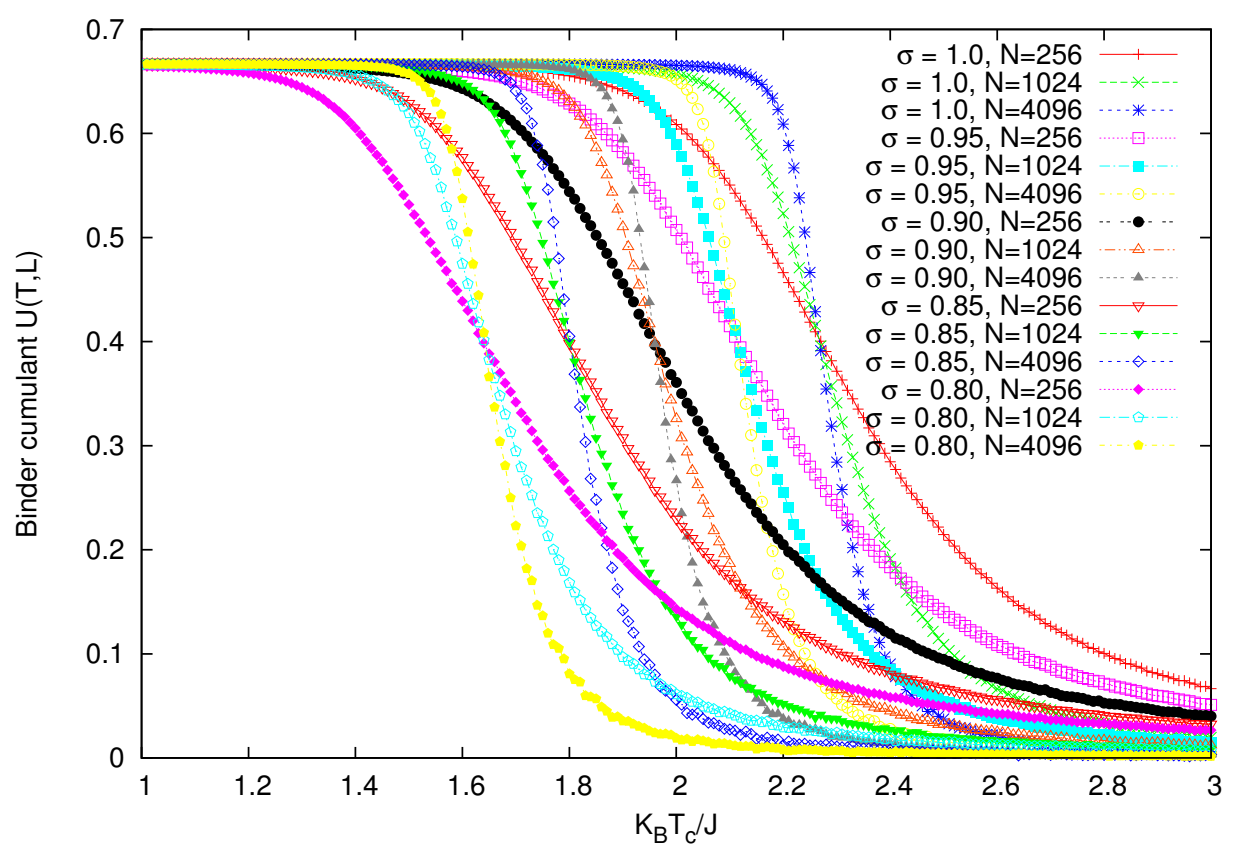

Fig. 3. The Binder cumulant $U(T, L)$ versus the temperature

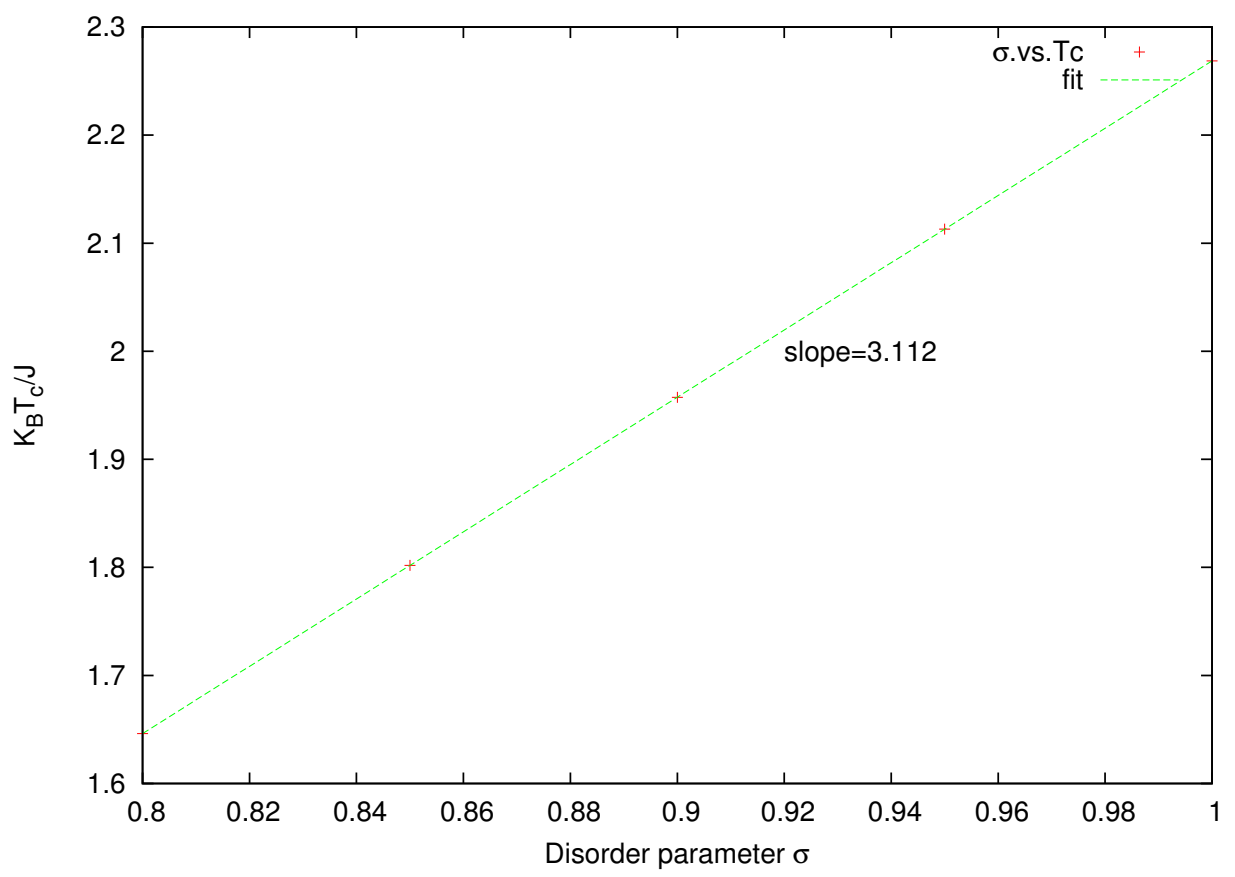

Fig. 4. The variation of the critical temperature relative to the disorder parameter $\sigma$

The slope of the $T_{c}(\sigma)$ line is found to be $3.112 \pm 0.023$ relative to that determinated by Harris [13] which is 3.016 and for Jayaprakash [12] is 3.108. This value of the slope is calculated near the 
bond concentration $\sigma=1.0$. Domany [14] has determinated the exact value of the slope near $\sigma=0.5$ (critical bond concentration) which is equal to 3.145 .

\section{Conclusion}

We have performed the Monte Carlo study for the bond diluted Ising model on the square lattice. The obtained results are in agreement with previous works [2], [3]. At this point, the effect of the free boundary conditions does not seem to affect the value of the critical temperature $T_{c}$. Further investigations are being done with larger system sizes and larger samples to get more precision in order to settle this issue.

\section{References}

1. L. Onsager, Physical Review 65, (1944) 117.

2. D. Zobin, Physical Review B 18, (1978) 2387.

3. Ioannis A. Hadjiagapiou, Physica A 390, (2011) 2229.

4. L. F. da Silva et all, J.Phys, Condens.Matter 21, (2009) 346005.

5. Ioannis A. Hadjiagapiou, Physica A 389, (2010) 3945.

6. S. Davatolhagh, M.Moshfeghian, Physica A 389, (2010) 3349.

7. ZHANG, X, NOVOTNY, M. A, Braz. J. Phys36, (2006) 664.

8. Ioannis A. Hadjiagapiou, Physica A 387, (2008) 2256.

9. K. Binder, Zeitschrift für Physik B Condensed Matter 43, (1981) 119.

10. Ioannis A. Hadjiagapiou, Physica A 390, (2011) 1279.

11. D. P. Landau and K. Binder, Monte Carlo Simulations in Statistical Physics (Cambridge: University Press, 2000).

12. C. Jayaprakash, E. K. Riedel, M. Wortis, Phys.Rev B 18, (1978) 2244.

13. A. B. Harris, J. Phys. C 7, (1974) 1671.

14. E. Domany, J. Phys. C 11, (1978) L337. 